\title{
A quitosana como biomaterial odontológico: estado da arte
}

\author{
Freni Kekhasharú Tavaria*, Eduardo Manuel Costa, Irene Pina-Vaz, Manuel Fontes Carvalho, \\ Maria Manuela Pintado
}

Resumo A impossibilidade de erradicar completamente os microrganismos da cavidade oral ou dos canais radiculares estimula a procura de novos materiais que permitam cada vez mais eficazmente, controlar ou prevenir o aparecimento de infeções. A quitosana é um biopolímero com uma estrutura química e características únicas que permitem que apresente excelente biocompatibilidade e que seja facilmente processado. A panóplia de aplicações é tão fascinante como ainda mal conhecida. A constatação do potencial e do valor industrial deste biopolímero conduziu à sua utilização em muitas aplicações de interesse tecnológico e, de uma forma acentuada, na área biomédica. As suas propriedades anti-oxidantes, anti-microbianas, anti-inflamatórias, cicatrizante e de inibição da formação de biofilmes tornam a sua aplicação na odontologia particularmente interessante. Com este trabalho pretendeu-se rever a literatura existente sobre aplicações da quitosana na área odontológica.

Palavras-chave Infecção oral, Ação antimicrobiana, Quitosana, Aplicações orais, Odontologia.

\section{Chitosan as a dental biomaterial: state of the art}

\begin{abstract}
The impossibility to completely eradicate the microorganisms from the oral cavity or from root canals has stimulated the search for new materials that efficiently prevent infections. Chitosan is a biopolymer with a unique chemical structure that renders it with outstanding biocompatibility and easy processing characteristics. The array of applications available is fascinating, as well as poorly understood. The industrial possibilities led to its use in several applications of varied technological interests, and especially in the biomedical area. Furthermore, its anti-oxidant, anti-microbial, anti-inflammatory, wound healing and biofilm inhibiting properties make it especially attractive to be used in the odontological arena. In this review, we address the state of the art on chitosan-based applications for odontology.
\end{abstract}

Keywords Oral infection, Anti-microbial activity, Chitosan, Oral applications, Odontology. 


\section{Introdução}

A cavidade oral é um dos locais com maior densidade microbiana do corpo humano de onde já foram isoladas mais de 500 espécies utilizando técnicas de identificação molecular (Takahashi, 2005). Estes microrganismos colonizam a superfície oral onde formam consórcios microbianos referidos como "placa bacteriana" ou "biofilme". A diversidade abiótica (microambientes) da cavidade permite o estabelecimento de diferentes comunidades microbianas formando as placas supragengival, subgengival e lingual.

O motivo mais frequente de consulta e intervenção odontológica são as infeções orais. O primeiro passo no estabelecimento da infeção oral é a adesão do microrganismo às células do hospedeiro, ou seja, a formação do biofilme (Sutherland, 2001). Este complexo ecossistema microbiano, bem estruturado e altamente dinâmico, facilita a obtenção de nutrientes e a manutenção de um ambiente com condições físicas e químicas adequadas (Agnelli, 2011). Uma vez estabelecidos, os biofilmes mantêm-se relativamente estáveis sobrevivendo a alterações frequentes no meio ambiente que os rodeia, mas quando ocorrem alterações na sua ecologia, levando à predominância de uma ou mais estirpes relativamente a outras, podem induzir o aparecimento de doenças orais.

A preparação químico mecânica é, sem dúvida, de importância singular para o sucesso do tratamento endodôntico. No entanto, este fato não diminui a importância de uma boa obturação que visa o selamento de vestígios de irritantes no sistema canalar e a minimização dos efeitos nocivos de eventuais derrames apicais e coronais. Porque atualmente não existem cimentos nem técnicas que garantam o selamento adequado de todo o sistema canalar, o cimento deve, pelo menos, reduzir o esvazamento e aprisionar os microrganismos remanescentes, de forma a evitar o seu acesso aos tecidos periradiculares (Siqueira et al., 2000). Por isso é recomendado o uso de medicação intracanalar como derivados fenólicos, depósitos de clorohexidina, soluções de iodo/iodeto de potássio e hidróxido de cálcio (Morrier et al., 2003). $\mathrm{O} \mathrm{Ca}(\mathrm{OH})_{2}$ é uma escolha popular dada a sua natureza alcalina que lhe confere atividade anti-microbiana e o seu papel vital na iniciação do processo de remineralização óssea. No entanto, alguns estudos (Doyon e Dumsha, 2005) referem que a exposição prolongada (mais de 180 dias) da dentina radicular ao hidróxido de cálcio pode afetar as suas características físicas, com possíveis implicações clínicas para os dentes tratados, como maior frequência de fraturas a longo prazo (Andreasen et al., 2002).
Os materiais obturadores de canais radiculares podem ser sólidos ou plásticos. Nos primeiros incluem-se os cones de prata e de gutta-percha, enquanto no segundo tipo estão incluídas as pastas e os cimentos. A gutta-percha na forma de cones é o material obturador mais utilizado e com mais sucesso (Gutmann e Witherspoon, 2000) uma vez que é bem tolerado pelos tecidos vivos e apresenta facilidade no seu manuseio independentemente da técnica utilizada. A gutta-percha é o trans-1,4-poliisopreno obtido pela coagulação do látex produzido pelas árvores da família Sapotaceae. A gutta é rígida à temperatura ambiente, tornando-se maleável a $25-30^{\circ} \mathrm{C}$, amolece a $60^{\circ} \mathrm{C}$ e derrete a $100^{\circ} \mathrm{C}$ com decomposição parcial (Maniglia-Ferreira et al., 2005). Na realidade, a gutta-percha é apenas um dos constituintes dos cones dentários. Estes possuem também na sua composição óxido de zinco, sulfato de bário (ou de outros metais), ceras e resinas. A percentagem destes constituintes varia de acordo com a marca comercial influenciando como é óbvio, as suas propriedades físico-químicas, bem como o seu desempenho biológico.

A variedade de materiais obturadores plásticos existentes é imensa. São exemplos mais típicos o cimento de fosfato de zinco, de policarboxilato, de fosfato de cálcio, de óxido de zinco e eugenol, de ionômeros de vidro e, o cimento de ionômero de vidro modificado por resina. Todos estes materiais diferem na sua composição química e, portanto, apresentam características físicas, mecânicas e biológicas diferentes. Vários estudos estão documentados, nos quais se fez a avaliação da eficácia de diferentes cimentos contra os diferentes microrganismos da flora endodôntica, cariogênica ou da saliva, e os resultados claramente mostram a falta dum padrão de resposta ao tratamento (Haapasalo et al., 2007; Melker et al., 2006; Siqueira e Rôças, 2008).

Apesar da terapia endodôntica ter como objetivo fundamental a limpeza e eliminação de microrganismos no sistema de canais radiculares, através de uma correta instrumentação e desinfeção do sistema para possibilitar que os tecidos envolvidos retornem ao seu estado normal, algumas vezes o dentista depara-se posteriormente com sinais e sintomas de uma infeção secundária (Dotto et al., 2006). A impossibilidade de erradicar completamente os microrganismos da cavidade oral ou dos canais radiculares tem motivado a busca de novos materiais que permitam, cada vez mais eficazmente, prevenir o aparecimento da re-infeção oral. $\mathrm{O}$ uso de biomateriais artificiais no tratamento de tecidos doentes foi iniciado na área da dentisteria. Os avanços científicos e tecnológicos na área de biomateriais e dispositivos médicos permitiram um desenvolvimento acentuado nesta área, em particular, 
focando-se em novas biomacromoléculas e materiais biocompatíveis para uso clínico (Ji et al., 2009).

Nesse âmbito, a quitosana, um polissacárido proveniente da desacetilação da quitina, obtida essencialmente da carapaça de caranguejo e camarão apresenta-se como uma nova possibilidade. Como macromolécula não tóxica, biocompatível e com comprovadas propriedades anti-inflamatória e cicatrizante (Tavaria et al., 2009), o interesse por este biopolímero tem vindo a aumentar. Nos últimos anos, a quitosana tem sido vastamente utilizada na veiculação de medicamentos, bem como na engenharia de tecidos aplicada à odontologia. A possibilidade de a incluir em formulações de cimentos dentários, colutórios e cones tem sido avaliada mais recentemente. Neste contexto, o objetivo deste trabalho foi o de rever a literatura existente em relação a aplicações com base na quitosana para a odontologia, publicados nos últimos anos.

\section{A Quitosana: Definição}

A quitosana é um biopolímero com uma estrutura química única formando um policátion linear, com elevada densidade de carga e de grupos reativos assim como inúmeras ligações de hidrogénio. Estas características permitem que exiba uma excelente biocompatibilidade e que seja facilmente processado. O termo "quitosana" refere-se a um grupo heterogêneo de polímeros que combinam uma vasta gama de características físico-químicas e biológicas, e que permitem uma panóplia de aplicações tão fascinantes como ainda mal conhecidas (Kumar, 2000). A constatação deste potencial e do valor industrial deste biopolímero conduziu à sua utilização em muitas aplicações de interesse tecnológico e, de uma forma acentuada, na área biomédica.

A quitosana é primariamente produzida a partir da quitina, que existe abundantemente na natureza como componente estrutural do exosqueleto de artrópodes (incluindo crustáceos e insetos), diatomáceas, algas e paredes celulares de alguns fungos. Estruturalmente a quitina é um mucopolissacárido linear e insolúvel em água. Esta estrutura é muito semelhante à da celulose onde o grupo hidroxila [-OH] da posição $\mathrm{C} 2$ é substituído pela acetamida [- $\left.\mathrm{NHCOCH}_{3}\right]($ Kumar, 2000). As fontes de quitina para processamento industrial são as cascas de crustáceos e os micélios fúngicos (Kumar et al., 2005). A sua extração envolve a remoção ácida do carbonato de cálcio (desmineralização) e a desproteinização em solução básica quente (normalmente hidróxido de $\mathrm{Na}$ ou $\mathrm{K}$ ) (Kumar, 2000). A quitina é, no entanto pouco solúvel e pouco reativa. A sua estrutura pode ser modificada por remoção dos grupos acetila que se encontram ligados a radicais amina na posição $\mathrm{C} 2$ do anel de glucano por hidrólise química produzindo um derivado desacetilado. Quando a fração de grupos amina acetilados é reduzida para 35-40\%, o co-polímero resultante é denominado de quitosana. A quitosana é assim caraterizada pelo seu peso molecular (MW) e grau de acetilação (DA). A quitosana disponível comercialmente tem grau de desacetilação $>85 \%$ (DA $<15 \%$ ), e peso molecular compreendido entre 100-1000 kDa. É geralmente aceito que a quitosana de baixo peso tem $\mathrm{MW}<50 \mathrm{kDa}$, a de médio $50-150 \mathrm{kDa}$ e a de alto peso $>150 \mathrm{kDa}$. A quitosana é uma base fraca insolúvel em água, mas solúvel em soluções ácidas diluídas abaixo do seu $\mathrm{pKa}(6,3)$, onde as unidades de glucosamina $\left(-\mathrm{NH}_{2}\right)$ podem ser convertidas na sua forma protonada $\mathrm{e}$ solúvel $\left(\mathrm{NH}_{3}^{+}\right)$. A solubilidade da quitosana depende da sua origem biológica, peso molecular e grau de acetilação (Goy et al., 2009) e isso afeta diretamente as aplicações a que pode ser sujeita.

\section{Propriedades Relevantes para a Odontologia}

Dentre as inúmeras propriedades biológicas descritas para a quitosana, a capacidade antioxidante, antimicrobiana, inibição da formação de biofilmes, anti-inflamatória e cicatrizante são talvez as mais relevantes para a odontologia.

\section{Antioxidante}

O stress oxidativo na cavidade oral nasce da resposta anti-inflamatória resultante de infeções periodontais. Isto se deve à liberação de ácido hipoclórico e oxigênio livre no fluido crevicular por parte de leucócitos polimorfonucleares (PMN), em resposta à infeção por patógenos periodontais (Petti e Scully, 2009). A resposta do organismo a este stress oxidativo faz-se através da libertação de sequestradores enzimáticos extracelulares, que assim repôem o equilibrio (Chapple et al., 2007). No entanto, em casos de desequilíbrio, este stress oxidativo resulta no esgotamento dos sequestradores levando a atividade antioxidante total para valores nulos o que pode originar a destruição do tecido periodontal (Chapple et al., 2007; Petti e Scully, 2009). A concentração de antioxidantes extracelulares é elevada em pacientes sem periodontite e concentrações elevadas estão associadas a um menor risco de desenvolvimento de periodontite crônica. Inversamente, em casos de níveis de antioxidantes extracelulares extremamente baixos, o risco de desenvolvimento de periodontite crônica é elevado (Chapple et al., 2007). Foi sugerido que nestes casos uma dieta rica em antioxidantes pode contribuir 
para o aumento da atividade antioxidante dos fluidos orais. A administração de polifenóis através de chá verde aumenta a capacidade antioxidante da saliva contribuindo assim decisivamente para o equilíbrio entre antioxidantes e oxidantes no fluido crevicular (Petti e Scully, 2009) prevenindo assim a periodontite e a cárie dentária.

Atualmente já existem vários estudos relativos à atividade antioxidante da quitosana tendo Wenjun et al. (2002) determinado o mecanismo responsável por esta atividade. $\mathrm{Na} 1^{\mathrm{a}}$ etapa, os radicais hidroxila subtraem átomos de hidrogênio à posição 5 da quitosana levando à formação de radicais hidroxi-metila. Subsequentemente, os radicais hidroxi-metila reagem entre si terminando assim a reação em cadeia. Adicionalmente, a presença de grupos amina na estrutura da quitosana confere-lhe uma forte capacidade de quelação do $\mathrm{Cu}^{2+}$, inibindo assim a formação de radicais hidroxila resultantes de reações de Fenton. Isto explica o porquê da porcentagem de inibição ser proporcional ao grau de desacetilação, pois quanto maior for o grau de desacetilação mais grupos amina estarão disponíveis para reagir. Assim sendo, é possível concluir que a capacidade de sequestro ("scavenging") de radicais livres e a resultante atividade antioxidante da quitosana estão ligadas aos grupos hidroxila e amina da cadeia polimérica, e que a quantidade destes grupos existentes na cadeia afetam a atividade antioxidante da quitosana (Sun et al., 2007; Wenjun et al., 2002).

Relativamente ao peso molecular da quitosana, verificou-se uma relação inversa com a ação antioxidante; quitosanas de baixo peso molecular apresentam maiores porcentagens de atividade antioxidante total quando comparadas com as de alto peso molecular (Xing et al., 2005). Isto se deve ao fato das cadeias de quitosana de baixo peso molecular serem mais lineares (menos emaranhadas) do que as de alto peso molecular, permitindo assim, um acesso mais fácil dos componentes ativos da cadeia aos radicais livres em solução (Wenjun et al., 2002).

Os radicais livres afetados pela atividade antioxidante da quitosana já foram descritos em vários estudos. Xing et al. (2005) demonstraram a atividade antioxidante da quitosana e seus derivados relativamente ao radical hidroxila e ao ânion superóxido. Wenjun et al. (2002), por outro lado, demonstraram a atividade relativamente a radicais lipídicos de ácido linoleico, a produtos de peroxidação lipídica do soro de rato e a ânions superóxido. Sun et al. (2007) confirmaram os resultados já referidos ao provar a existência de atividade antioxidante de oligômeros de quitosana relativamente aos ânions superóxido.

\section{Antimicrobiana}

A quitina e a quitosana têm sido investigados como agentes antimicrobianos contra uma vasta gama de microganismos-alvo como algas, bactérias, leveduras e fungos em experiências in vivo e in vitro envolvendo a quitosana em diversas formas (soluções, filmes e compósitos). Nestes estudos (Costa et al., 2012; Elsaka e Elnaghy, 2012; Íkinci et al., 2002), a quitosana foi considerada como tendo atividade bactericida ou bacteriostática, frequentemente sem distinção entre estas atividades. Dados recentes da literatura caraterizam a quitosana como sendo mais bacteriostática do que bactericida (Coma et al., 2002), apesar do mecanismo exato não ser ainda exatamente conhecido e a ação antibacteriana estar dependente da interação de diferentes fatores (Raafat et al., 2008).

$\mathrm{O}$ espectro de ação da quitosana abrange fungos filamentosos, leveduras e bactérias, sendo, no entanto, mais eficaz contra bactérias gram-positivas (Jeon et al., 2001; No et al., 2002; Rhoades e Roller, 2000). A quitosana apresenta-se extremamente promissora na área da odontologia uma vez que mostra uma forte ação na redução da placa dentária, assim como comprovada ação antimicrobiana in vitro contra vários patógenos da cavidade oral diretamente implicados na formação da placa e na periodontite, tais como o Actinobacillus actinomycetemcomitans, o Streptococcus mutans e a Porphyromonas gingivalis (Choi et al., 2001; Íkinci et al., 2002). No entanto, sendo a sua ação essencialmente bacteriostática, pode conduzir ao aparecimento de sub-populações mais resistentes após a adaptação fisiológica das células ao stress da presença da quitosana (Raafat et al., 2008). Três modelos têm sido propostos para a sua ação antimicrobiana, sendo a interação das cargas positivas das moléculas de quitina/quitosana com as cargas negativas da membrana microbiana a mais aceitável. Neste modelo a interação é mediada pelas forças eletrostáticas entre o grupo protonado $\mathrm{NH}_{3}{ }^{+} \mathrm{e}$ os resíduos carregados negativamente, presumivelmente competindo com os íons $\mathrm{Ca}^{2+}$ pelos locais eletronegativos na superfície da membrana (Rabea et al., 2003).

Os estudos referentes à capacidade anti-microbiana da quitosana e dos seus derivados, extraídos de diversas fontes e sob diferentes condições, são inúmeros. $\mathrm{Na}$ maior parte dos casos existem discrepâncias enormes nos resultados obtidos, o que não é surpreendente dado que a atividade da quitosana 'in vitro' é dependente de muitos fatores intrínsecos e extrínsecos, diretamente relacionados ao peso molecular, grau de desacetilação, viscosidade, solventes e concentração ou com factores abióticos como a estirpe microbiana em questão e seu estado fisiológico, o meio de cultura, $\mathrm{pH}$, temperatura, força iônica, e presença de íons metálicos, EDTA e matéria orgânica (Cheng et al., 2003; Cho et al., 2006; Chung et al., 2003). 


\section{Inibição de biofilmes}

Em termos da influência da quitosana na capacidade de adesão de estirpes orais o estudo de Tarsi et al. (1997) demonstrou que a quitosana consegue influenciar a adesão de $S$. mutans. Neste estudo foi testada a capacidade de uma quitosana de baixo peso molecular e de dois seus derivados $(N$-carboximetil quitosana e imidazolil quitosana) em prevenir a adesão de $S$. mutans à hidroxiapatita (HA). Os resultados mostraram que a presença de concentrações sub-letais de quitosana pôde prevenir a ligação do $S$. mutans à HA confirmando assim a possibilidade de utilização da quitosana como agente preventivo da formação de biofilmes. Adicionalmente, no caso de infeções resistentes ao tratamento endodôntico convencional, nas quais têm sido implicados microrganismos como o Enterococcus faecalis e a Candida albicans, e a habitual utilização do hidróxido de cálcio não tem mostrado a eficácia habitual (Cwikla et al., 2005), a adição de quitosana parece inibir a formação de biofilmes potencializando assim a ação antimicrobiana do $\mathrm{Ca}(\mathrm{OH})_{2}$.

\section{Anti-inflamatória}

A inflamação crônica é uma resposta patológica, de longo prazo que envolve o dano do próprio tecido por metaloproteinases da matriz (Hu et al., 2007). Esta está relacionada com algumas doenças entre as quais com a doença periodontal. A quitosana e respetivos oligômeros inibem a ativação e a expressão da metaloproteinase-2 da matriz em fibroblastos humanos (Kim e Kim, 2006), estimulando a cicatrização dos tecidos. O efeito imunoestimulador de derivados de quitosana tem sido atribuído à presença dos grupos $N$-acetil-D-glucosamina (Porporatto et al., 2003), que atuam estimulando as células inflamatórias como os neutrófilos PMN, macrófagos e fibroblastos. A quitosana promove a produção do fator de transformação do crescimento $\beta$ (TGF- $\beta$ ) e do fator de crescimento derivado de plaquetas por monócitos humanos derivados de macrófagos (Ueno et al., 2001a) envolvidos na resposta inflamatória. A quitosana de alto peso aumenta a produção de interleucina-1 (IL-1), fator de necrose tumoral alfa (TNF- $\alpha$ ), fator estimulante de colônias de granulócitos e de macrófagos (GM-CSF), óxido nítrico (NO) e interleucina-6 (IL-6) em macrófagos, contribuindo para a ação anti-inflamatória e anti-tumoral. Por outro lado, os oligômeros de quitosana estimulam a libertação de TNF- $\alpha$ e de IL-1 $\beta$, salientando o seu efeito imunoestimulador.

\section{Cicatrizante}

Adicionalmente, a propriedade imunomoduladora da quitosana advém da sua capacidade de estimular os macrófagos, que libertam a IL-1 o que, por sua vez, estimula a proliferação de fibroblastos e influencia a estrutura do colágeno. Os oligômeros de quitina e quitosana de baixo peso libertam também $N$-acetilglicosaminidase, que hidroliza a quitosana em unidades necessárias à biossíntese do ácido hialurónico e outros componentes da matriz extracelular (Silva et al., 2006), conduzindo à cicatrização. Feridas tratadas com quitosana mostram sinais de regeneração histológica como uma infiltração profusa de leucócitos PMN e maiores quantidades de colágeno e de osteopontina no local (Ueno et al., 2001b). A capacidade cicatrizante é maior para a quitosana do que para a quitina; quanto maior o grau de desacetilação, maior a resistência das feridas à ruptura e mais fibroblastos ativos aparecem no local (Minagawa et al., 2007).

\section{Quitosana: Potencial de Aplicação}

Apesar de muitos recursos terem sido postos no desenvolvimento da investigação e de processos e aplicações para a quitosana, apenas nas duas últimas décadas estes têm sido direcionados para a sua comercialização assim como dos seus derivados. As principais áreas de aplicação da quitosana são: agricultura (mecanismos defensivos e adubo para plantas), tratamento de águas (floculante para clarificação, remoção de íons metálicos, polímero ecológico e remoção de odores), indústria alimentar (fibras dietéticas, redutor de colesterol, conservante, fungicida e bactericida, revestimento para frutos), cosmética (exfoliante para a pele, tratamento do acne, hidratante capilar, creme dental) e biofarmacêutica (imunológico, antitumoral, hemostático e anticoagulante). No entanto, a sua maior aplicação é na área biomédica (suturas cirúrgicas, implantes dentários, reconstituição óssea, lentes de contato, liberação controlada da medicamentos, encapsulamento de materiais) (Azevedo et al., 2007).

Segundo Decker et al. (2005), as aplicações da quitosana e seus derivados na área da odontologia, devem-se essencialmente às suas propriedades bioadesivas, viscosas, permeabilizantes, antimicrobianas e anticariogênicas que promovem a liberação prolongada de medicamentos na cavidade oral mostrando eficácia e potencial no tratamento de doenças periodônticas/candidíases orais/mobilidade dentária e na redução da placa dentária. Atualmente existem já diversas aplicações da quitosana na odontologia (Tabela 1). Vários estudos comprovam a eficácia da quitosana in vitro bem como das suas aplicações. Em 2006, Azcurra et al. mostraram que a quitosana de alto peso era eficaz na redução da hidrofobicidade e adesão de Candida albicans a células dos tecidos moles da cavidade oral. Géis de quitosana são menos ativos 
Tabela 1. Produtos já existentes no mercado à base de quitosana.

Table 1. Commercially available chitosan-based products.

\begin{tabular}{|c|c|c|}
\hline Produto & Ação proposta & Referência(s) \\
\hline Gel de quitosana & Tratamento de periodontite crónica & $\begin{array}{l}\text { Akınciba1 et al. (2007); Boynueğri et al. } \\
\text { (2009) }\end{array}$ \\
\hline Hidrogéis & Tratamento de aftas & Pignatello et al. (2009) \\
\hline Filmes & Atividade antifúngica & Şenel et al. (2000) \\
\hline Micropartículas quitosana/Flúor & $\begin{array}{c}\text { Aumentar a absorção de flúor, proteção } \\
\text { a cáries }\end{array}$ & Keegan et al. (2012) \\
\hline Membranas & Regeneração tecidular, periodontite & Ho et al. (2010) \\
\hline Cimentos endodônticos & $\begin{array}{c}\text { Regeneração óssea e diminuição da } \\
\text { inflamação }\end{array}$ & Li et al. (2009) \\
\hline Colutórios & $\begin{array}{c}\text { Redução da placa bacteriana e do } \\
\text { número de } S \text {. mutans }\end{array}$ & $\begin{array}{l}\text { Sano et al. }(2002,2003) \\
\text { Bae et al. }(2006)\end{array}$ \\
\hline Cones & $\begin{array}{c}\text { Material de obturação dos canais } \\
\text { radiculares }\end{array}$ & Jia e Alpert (2007) \\
\hline \multirow[t]{2}{*}{ Dentífrico } & Efeito antimicrobiano sobre biofilme oral & $\begin{array}{l}\text { Efstratiou et al. (2007); Verkaik et al. } \\
\qquad \text { (2011) }\end{array}$ \\
\hline & $\begin{array}{c}\text { Redução da descalcificação à volta de } \\
\text { braquetes dentários }\end{array}$ & Uysal et al. (2011) \\
\hline Chip dentário & Tratamento da periodontite & Jothi et al. (2009) \\
\hline Pastilha elástica & $\begin{array}{c}\text { Redução do número de } S \text {. mutans na } \\
\text { cavidade oral } \\
\text { Efeito antimicrobiano }\end{array}$ & $\begin{array}{c}\text { Hayashi et al. }(2007 \mathrm{a}, 2007 \mathrm{~b}) \\
\text { Miao et al. }(2009)\end{array}$ \\
\hline
\end{tabular}

contra a Candida albicans do que a clorohexidina; no entanto a atividade antifúngica da clorohexidina aumenta com a acrescida concentração de quitosana no gel. A incorporação de baixas concentrações de clorohexidina $(0,1 \%)$ a géis de quitosana a $2 \%$ é eficaz no tratamento de candidíases da cavidade oral (Şenel et al., 2000); o uso de formulações de quitosana em filmes tem liberação prolongada permanecendo intactos por 4 horas, o que pode ser vantajoso no tratamento da periodontite. Este fato é reforçado pela comprovada ação sobre os patógenos periodontais Porphyromonas gingivalis (Íkinci et al., 2002) e Actinobacillus actinomycetemcomitans (Choi et al., 2001) por erupção da membrana bacteriana. Por outro lado, a inclusão de um derivado de quitosana (carboximetil quitosana quaternária) ao hidróxido de cálcio para recobrimento pulpar induziu a formação de dentina e foi mais eficaz do que o tratamento apenas com o hidróxido de cálcio (Sun et al., 2006).

Entre as aplicações encontram-se géis para o tratamento da periodontite crônica (Akıncibay et al., 2007; Boynueğri et al., 2009) demonstrando que o uso destes géis na regeneração periodôntica promove o preenchimento ósseo, quer isoladamente quer quando combinado com a matriz óssea desmineralizada. Existem também hidrogéis com quitosana para aplicação bucal de anestéticos no tratamento de aftas; estes mostraram-se eficazes promovendo a liberação local e o aumento da biodisponibilidade do componente ativo, com ampla duração da ação reduzindo o número de aplicações (Pignatello et al., 2009). Recentemente, foram produzidas micropartículas de quitosana/flúor por atomização (Keegan et al., 2012), e os autores sugerem que estas poderão ser usadas para aumentar a absorção de flúor dos produtos de higiene oral, potencializando a proteção contra cáries.

Mais ainda, a adição de quitosana num cimento de $\mathrm{ZnO}$ e nano-hidroxiapatita potencializou a regeneração óssea e diminuiu a inflamação (Li et al., 2009). No entanto, um estudo anterior (Kishen et al., 2008) havia mostrado que a incorporação de nanopartículas de quitosana a cimentos dentários não altera a permeabilidade destes, mas melhora significativamente as suas propriedades anti-microbianas e a capacidade de lixiviação dos componentes antibacterianos. A inclusão da quitosana em colutórios, para redução da placa bacteriana também tem sido objeto de estudo de vários investigadores; aqui é de salientar o estudo de Sano et al. (2003), que concluíram que, num espaço de tempo limitado (14 dias), um colutório à base de quitosana foi eficaz na redução da placa e na contagem de Streptococcus mutans da saliva. A ação anti-placa da quitosana foi também avaliada num estudo anterior (2002) por Sano et al., que concluíram que a quitosana com um peso molecular $>5-6 \mathrm{kDa}$ e um grau de desacetilação de $50-60 \%$ era eficiente como agente anti-placa devido às suas propriedades policatiônicas. A inibição no resurgimento da placa 
dentária após administração de colutórios contendo quitosana também foi avaliada por Bae et al. (2006), que sugerem que o mecanismo de ação deverá consistir na inativação enzimática, quelação de íons metálicos essenciais e na formação de complexos polieletrolíticos com os componentes da superfície bacteriana. A adição de quitosana a pastas dentífricas como agente gelificante reduziu em $70 \%$ a placa bacteriana e em $85 \%$ as contagens bacterianas (Mohire e Yadav, 2010) preenchendo a maioria dos critérios estéticos e médicos para um produto de higiene oral. Verkaik et al. (2011) também adicionaram quitosana a pasta de dentes contendo extratos de plantas e obtiveram uma eficácia comparável à da ciclohexidina, não apenas na sua ação imediata, como também numa perspetiva mais duradoura. Estudos recentes (Uysal et al., 2011) mostraram que dentífricios à base de quitosana podem também reduzir a descalcificação do esmalte à volta dos braquetes dentários em pacientes em reabilitação e com fraca higiene oral. Foi também avaliada a eficácia de um chip dentário contendo clorohexidina numa base de quitosana no tratamento da periodontite, e este mostrou-se clinicamente seguro e uma opção de tratamento eficaz (Jothi et al., 2009), pois permitiu reduzir a profundidade de sondagem aumentando o ganho de aderência clínica. Este trabalho sugere esta opção como alternativa na gestão de pacientes com periodontite crônica, especialmente durante a manutenção intercalar entre tratamentos. Por fim, pastilhas elásticas com quitosana foram também produzidas, e comprovadamente reduziram o número de bactérias cariogênicas na cavidade oral (saliva), em especial o Streptococcus mutans (Hayashi et al., 2007a, 2007b; Miao et al., 2009).

Apesar das inúmeras aplicações já existentes para a quitosana em produtos orais, outros usos poderão incluir a sua utilização em materiais onde a interação com outras substâncias seja potencializada. Uma delas seria a sua inclusão em gutta-percha, cimentos e filmes para limpeza dentária. O uso de derivados de quitosana (quitosana funcionalizada) em aplicações orais também pode constituir um benefício adicional, pois a funcionalização não só aumenta a solubilidade como diminui a potencial toxicidade.

É ainda de salientar o uso de revestimentos de quitosana para a veiculação local de antimicrobianos (como a tetraciclina ou a clorohexidina) na mucosa oral, canais radiculares ou bolsas periodontais, sem toxicidade para o hospedeiro (Decker et al., 2005). Como material seletivamente permeável, a quitosana é um bom candidato para este fim, potencializado pela sua capacidade de também promover a regeneração óssea (Spin-Neto et al., 2008).

\section{Limitações do Uso da Quitosana}

Apesar das inúmeras vantagens associadas à quitosana terem conduzido ao crescente número de aplicações, existem algumas limitações associadas ao seu uso. No caso de aplicações biomédicas, questões relacionadas com a sua segurança, nomeadamente com a sua genotoxicidade, têm sido alvo de contestação e de investigação. Os dados presentes na literatura a este respeito são limitados. Oligômeros de quitosana (produzidos por despolimerização da quitosana) não mostraram indícios de mutagenicidade nem de genotoxicidade no teste de Ames, micronúcleo e no teste de alteração de esperma quando administrados oralmente (Qin et al., 2006). Ishihara et al. (2002) também reportaram ausência de mutagenicidade da quitosana reticulada ou em hidrogel sobre várias linhagens celulares. Ao avaliar a citocompatibilidade da quitosana e de um derivado (quaternário) usando o teste MTT e a microscopia eletrônica de transmissão, Ji et al. (2009), concluíram que os grupos quaternários no derivado em altas concentrações tornam o ambiente extremamente catiônico e produzem uma forte atração eletrostática com os grupos carregados negativamente na superfície de células do ligamento periodontal conduzindo à destruição das células, causando assim um efeito citotóxico acentuado. Também foi demonstrada citotoxicidade no trabalho de Opanasopit et al. (2007), que testaram sais de quitosana em células Caco-2, com valores de IC50 na gama de 0,22-0,72 mg/mL. Em 2005, Chae et al. já tinham estudado o efeito do peso molecular da quitosana na absorção oral utilizando também células Caco-2 e modelos de absorção intestinal em ratos, demonstrando que os seus oligômeros hidrosoluvéis podiam ser considerados seguros e potenciais candidatos para aplicações farmacêuticas e biomédicas.

Baldrick (2010) num estudo sobre o uso da quitosana como excipiente conclui que a administração oral parece segura uma vez que os dados toxicológicos indicam que altas doses são bem toleradas por ratos e coelhos, embora sem conhecimento exato do que ocorre sistemicamente. A ingestão oral por humanos através de suplementação dietética (em gramas) não apresentou efeitos adversos. A probabilidade de acumulação/retenção da quitosana que entra no organismo por absorção é também baixa uma vez que é convertida em derivados de glucosamina que serão naturalmente excretados ou usados como fonte de açúcares e aminoácidos. No entanto, os dados existentes atualmente não dão uma resposta clara quanto ao uso parenteral. Apesar da quitosana em condições adequadas apresentar uma baixa toxicidade, na gama das $\mu \mathrm{g} / \mathrm{mL}$, os seus derivados e produtos resultantes deverão ser avaliados individualmente (Kean e Thanou, 2010). 
Uma outra questão que limita a aplicação da quitosana prende-se à necessidade de esterilizar os produtos resultantes para uso parenteral ou para aplicações que envolvam contato com fluidos corporais. Os métodos de esterilização mais utilizados para produtos farmacêuticos e médicos são a exposição ao calor seco, esterilização com calor úmido (autoclavagem), óxido de etileno ou radiação gama (Khor e Lim, 2003). No entanto, antes do uso de qualquer destes métodos é necessário avaliar o seu efeito sobre as propriedades finais do polímero. Em 1999, Lim et al. avaliaram o efeito do calor seco, autoclavagem e radiação gama sobre membranas de quitosana e concluiram que o uso de radiação em condições de anoxia era o método mais eficaz de esterilização de produtos à base de quitosana. Estes resultados contrastavam com os de Rao e Sharma (1997) que recomendavam a esterilização com calor úmido. O efeito do armazenamento também necessita ser avaliado uma vez que afeta a integridade do polímero (Kam et al., 1999). Pela disparidade de resultados encontrados nos vários estudos, é evidente que a quitosana sofre alterações na sua estrutura e no grau de solubilidade quando exposta a temperaturas superiores a $120^{\circ} \mathrm{C}$, e que se degrada e perde viscosidade quando exposta à radiação gama, o que consequentemente altera as suas propriedades biológicas (Spin-Neto et al., 2008). Em última análise, torna-se imperativa a necessidade de mais estudos direcionados a esta temática para que a quitosana possa completamente satisfazer o seu papel inovador na prática clínica.

\section{Conclusões}

Os comprovados efeitos antioxidante, antimicrobiano, anti-inflamatório e cicatrizante da quitosana e a sua capacidade de inibição da formação de biofilmes poderão representar uma vantagem no tratamento de doenças associadas à cavidade oral. $\mathrm{O}$ uso de dentífricios, colutórios e materiais dentários contendo quitosana pode permitir reduzir o número de microrganismos presentes, atrasar a formação de biofilmes, bem como acelerar o processo de cicatrização e potencializar a atividade antimicrobiana de outros materiais. No entanto, o efeito da esterilização desses produtos sobre as propriedades finais do polímero e a toxicidade associada necessitam de ser cuidadosamente avaliadas uma vez que a alta temperatura, o processamento e até mesmo o armazenamento alteram as suas propriedades biológicas. Finalmente, estudos adicionais sobre a eficácia destes materiais "in vivo", bem como uma mais completa compreensão da interação da quitosana com outros componentes presentes em produtos dentários são necessários para melhor se avaliar a sua ação nesta área.

\section{Agradecimentos}

Os autores agradecem à Agência de Inovação (ADI, Portugal) e ao Quadro de Referência Estratégico Nacional (QREN, Portugal) através do projeto QUITORAL - Desenvolvimento de novas formulações de quitosana com aplicação em medicina oral (QRENADI3474) e à Fundação para a Ciência e Tecnologia (FCT, Portugal) através do projeto PEst-OE/EQB/ LA0016/2011 pelo financiamento proporcionado.

\section{Referências}

Agnelli P. Redução do biofilme de $S$. mutans sobre um material restaurador dentário modificado com nanopartículas de prata - estudo in vitro [tese]. São Paulo: Universidade Federal de São Carlos; 2011

Akıncıbay H, Şenel S, Ay ZY. Application of chitosan gel in the treatment of chronic periodontitis. Journal of Biomedical Materials Research Part B: Applied Biomaterials. 2007; 80:290-6. PMid:16767723. http:// dx.doi.org/10.1002/jbm.b.30596

Andreasen JO, Farik B, Munksgaard EC. Long-term calcium hydroxide as a root canal dressing may increase risk of root fracture. Endodontics and Dental Traumatology. 2002; 18:134-7. http://dx.doi.org/10.1034/ j.1600-9657.2002.00097.x

Azcurra AI, Barembaum SR, Bojanich MA, Calamari SE, Aguilar J, Battellino LJ, Dorronsoro ST. Efecto del quitosán de alto peso molecular y del alginato de sodio sobre la hidrofobicidad y adhesión de Candida albicans a células. Medicina Oral Patolologia Oral y Cirurgia Bucal. 2006; 11:E120-E5. PMid:16505787.

Azevedo VVC, Chaves SA, Bezerra DC, Lia Fook MV, Costa ACFM. Quitina e quitosana: aplicações como biomateriais. Revista Elêctronica de Materiais e Processos. 2007; 23:27-34.

Bae K, Jun EJ, Lee SM, Paik DI, Kim JB. Effect of water-soluble reduced chitosan on Streptococcus mutans, plaque regrowth and biofilm vitality. Clinical Oral Investigations. 2006; 10:102-7. PMid:16572330. http:// dx.doi.org/10.1007/s00784-006-0038-3

Baldrick P. The safety of chitosan as a pharmaceutical excepient. Regulatory Toxicology and Pharmacology. 2010; 56:290-9. PMid:19788905. http:// dx.doi.org/10.1016/j.yrtph.2009.09.015

Boynueğri D, Özcan G, Şenel S, Uç D, Uraz A, Öğüş E, ÇakıIc1 B, Karaduman B. Clinical and radiographic evaluations of chitosan gel in periodontal intraosseous defects: a pilot study. Journal of Biomedical Materials Research Part B: Applied Biomaterials. 2009; 90B:461-6. PMid:19788905. http://dx.doi.org/10.1016/j.yrtph.2009.09.015

Chae SY, Jang MK, Nah JW. Influence of molecular weight on oral absorption of water soluble chitosans. Journal of Controlled Release. 2005; 102:383-94. PMid:15653159. http://dx.doi.org/10.1016/j.jconrel.2004.10.012 
Chapple I, Milward M, Dietrich T. The prevalence of inflammatory periodontitis in negatively associated with serum antioxidant concentrations. Journal of Nutrition. 2007; 137:657-64. PMid:17311956.

Cheng H-Y, Yu R-C, Chou C-C. Increased acid tolerance of Escherichia coli $0157: \mathrm{H} 7$ as affected by acid adaptation time and conditions of acid challenge. Food Research International. 2003; 36:49-56. http://dx.doi.org/10.1016/ S0963-9969(02)00107-2

Cho J, Heuzey M-C, Bégin A, Carreau P. Viscoelastic properties of chitosan solutions: Effect of concentration and ionic strength. Journal of Food Engineering. 2006; 74:500-15. http://dx.doi.org/10.1016/j.jfoodeng.2005.01.047

Choi BK, Kim KY, Yoo YJ, Oh SJ, Choi JH, Kim CY. In vitro antimicrobial activity of a chitooligosaccharide mixture against Actinobacillus actinomycetemcomitans and Streptococcus mutans. International Journal of Antimicrobial Agents. 2001; 18:553-7. http://dx.doi.org/10.1016/ S0924-8579(01)00434-4

Chung Y-C, Wang H-L, Chen Y-M, Li S-L. Effect of abiotic factors on the antibacterial activity of chitosan against waterborne pathogens. Bioresource technology. 2003; 88:179-84.

Coma V, Martial-Gros A, Garreau S, Copinet A, Salin F, Deschamps A. Edible antimicrobial films based on chitosan matrix. Journal of Food Science. 2002; 67:1162-9. http:// dx.doi.org/10.1111/j.1365-2621.2002.tb09470.x

Costa EM, Silva S, Pina C, Tavaria FK, Pintado MM. Evaluation and insights into chitosan antimicrobial activity against anaerobic oral pathogens. Anaerobe. 2012; 18:305-9. PMid:22561525. http://dx.doi.org/10.1016/j. anaerobe.2012.04.009

Cwikla S, Bélanger M, Giguère S, Progulske-Fox A, Vertucci F. Dentinal tubule disinfection using three calcium hydroxide formulations. Journal of Endodontics. 2005; 31:50-2. PMid:15614007. http://dx.doi.org/10.1097/01. DON.0000134291.03828.D1

Decker EM, von Ohle C, Weiger R, Wiech I, Brecx M. A synergistic chlorohexidine/chitosan combination for improved antiplaque strategies. Journal of Periodontal Research. 2005; 40:373-7. PMid:16105089. http://dx.doi. org/10.1111/j.1600-0765.2005.00817.x

Doyon G, Dumsha, T. Fracture resistance of human root dentin exposed to intracanal calcium hydroxide. Journal of Endodontics. 2005; 31:895-7. PMid:16306826. http:// dx.doi.org/10.1097/01.don.0000194542.02521.af

Dotto R, Travassos R, Ferreira R, Santos R, Wagner M. Avaliação da ação antimicrobiana de diferentes medicações usadas em endodontia. Revista Odonto Ciência. 2006; 21:266-9.

Efstratiou M, Papaioannou W, Nakou M, Ktenas E, Vrotsos IA, Panis V. Contamination of a toothbrush with antibacterial properties by oral microorganisms. Journal of Dentistry. 2007; 35: 331-7. PMid:17118507. http://dx.doi. org/10.1016/j.jdent.2006.10.007

Elsaka SE, Elnaghy AM. Antibacterial activity of calcium hydroxide combined with chitosan solutions and the outcomes on the bond strength of RealSealer sealer to radicular dentin.
Journal of Biomedical Research. 2012; 26:193-9. http:// dx.doi.org/10.7555/JBR.26.20110136

Goy RC, Britto D, Assis OBG. A review of the antimicrobial activity of chitosan. Polímeros 2009; 3:1-7.

Gutmann J, Witherspoon D. Obturação do sistema de canais radiculares limpo e modelado. In: Cohen S, Burns R, editors. Caminhos da Polpa. Rio de Janeiro: Guanabara Koogan; 2000. p. 243-340.

Haapasalo M, Qian W, Portenier I, Waltimo T. Effects of dentin on the antimicrobial properties of endodontis medicaments. Journal of Endodontics. 2007; 33:917-25. PMid:17878075. http://dx.doi.org/10.1016/j.joen.2007.04.008 Hayashi Y, Ohara N, Ganno T, Yamaguchi K, Ishizaki T, Nakamura T, Sato M. Chewing chitosan-containing gum effectively inhibits growth of cariogenic bacteria. Archives of Oral Biology. 2007a; 52:290-4. Pmid:17112460. http:// dx.doi.org/10.1016/j.archoralbio.2006.10.004

Hayashi Y, Ohara N, Ganno T, Ishizaki T, Yanagiguchi K. Chitosan-containing gum chewing accelerates antibacterial effect with an increase in salivary secretion. Journal of Dentistry. 2007b; 35:871-4. PMid:17900783. http://dx.doi. org/10.1016/j.jdent.2007.08.004

Ho MH, Hsieh CC, Hsiao SW, Thien D. Fabrication of asymmetric chitosan GTR membranes for the treatment of periodontal disease. Carbohydrate Polymers. 2010; 79:955-63. http://dx.doi.org/10.1016/j.carbpol.2009.10.031

Hu J, Van den Steen P, Sang Q, Opdenakker G. Matrix metalloproteinase inhibitors as therapy for inflammatory and vascular diseases. Nature Reviews Drug Discovery. 2007; 6:480-98. PMid:17541420. http:// dx.doi.org/10.1038/nrd2308

Íkinci G, Şenel S, Akıncıbay H, Kaş S, Erciş S, Wilson CG, Hincal AA. Effect of chitosan on a periodontal pathogen Porphyromonas gingivalis. International Journal of Phamaceutics. 2002; 235:121-7. http://dx.doi.org/10.1016/ S0378-5173(01)00974-7

Ishihara M, Nakanishi K, Ono K, Sato M, Kikuchi M, Saito Y, Yura H, Matsui T, Hattori H, Uenoyama M, Kurita A. Photocrosslinkable chitosan as a dressing for wound occlusion and accelerator in healing process. Biomaterials. 2002; 23:833-40. http://dx.doi.org/10.1016/ S0142-9612(01)00189-2

Jeon YJ, Park PJ, Kim SK. Antimicrobial effect of chitooligosaccharides produced by bioreactor. Carbohydrate Polymers. 2001; 44:71-6. http://dx.doi.org/10.1016/ S0144-8617(00)00200-9

Ji QX, Zhong DY, Lü R, Zhang WQ, Deng J, Chen XG. In vitro evaluation of the biomedical properties of chitosan and quaternized chitosan for dental applications. Carbohydrate Research. 2009; 344:1297-302. PMid:19535044. http:// dx.doi.org/10.1016/j.carres.2009.05.014

Jia W, Alpert B. Dental filling material and endodontic post. US patent 2007/0131139A1. Wallingford. 2007 June 14.

Jothi MV, Bhat KM, Pratibha PK, Bhat GS. The evaluation of a biodegradable dental chip containing chlorhexidine in chitosan base as a targeted drug delivery in the management of chronic periodontitis in patients. Drug Development Research. 2009; 70:395-401. http://dx.doi.org/10.1002/ ddr.20316 
Kam HM, Khor E, Lim LY. Storage of partially deacetylated chitosan films. Journal of Biomedical Materials Research B: Applied Biomaterials. 1999; 48:881-8. http://dx.doi. org/10.1002/(SICI)1097-4636(1999)48:6<881::AID-JB $\mathrm{M} 18>3.0 . \mathrm{CO} ; 2-2$

Kean T, Thanou M. Biodegradation, biodistribution and toxicity of chitosan. Advanced Drug Delivery Reviews. 2010; 62:3-11. PMid:19800377. http://dx.doi. org/10.1016/j.addr.2009.09.004

Keegan GM, Smart JD, Ingram MJ, Barnes LM, Burnett GR, Rees GD. Chitosan microparticles for the controlled delivery of fluoride. Journal of Dentistry. 2012; 40:229-40. PMid:22212236. http://dx.doi.org/10.1016/j.jdent.2011.12.012

Khor E, Lim LY. Implantable applications of chitin and chitosan. Biomaterials. 2003; 24:2339-49. http://dx.doi. org/10.1016/S0142-9612(03)00026-7

Kim M, Kim S. Chitooligosaccharides inhibit activation and expression of matrix metalloproteinase- 2 in human dermal fibroblasts. FEBS Letters. 2006; 580:2661-6. Pmid:16647062. http://dx.doi.org/10.1016/j.febslet.2006.04.015

Kishen A, Shi Z, Shrestha A, Neoh KG. An investigation on the antibacterial and antibiofilm efficacy of cationic nanoparticulates for root canal disinfection. Journal of Endodontics. 2008; 34:1515-20. PMid:19026885. http:// dx.doi.org/10.1016/j.joen.2008.08.035

Kumar RMNV. A review of chitin and chitosan applications. Reactive and Functional Polymers. 2000; 46:1-27. http:// dx.doi.org/10.1016/S1381-5148(00)00038-9

Kumar ABV, Varadaraj MC, Gowda LR, Tharanathan $\mathrm{RN}$. Characterization of chitooligosaccharides prepared by chitosanolysis with the aid of papain and pronase, and their bactericidal action against Bacillus cereus and Escherichia coli. Biochemistry Journal. 2005; 391:167-75. PMid:15932346 PMCid:1276913. http://dx.doi.org/10.1042/ BJ20050093

Li Z, Yubao L, Yi Z, Lan W, Jansen J. In vitro and in vivo evaluation of the bioactivity of $\mathrm{ZnO}$ containing nano-hydroxyapatite/chitosan cement. Journal of Biomedical Materials Research Part A. 2009; 93:269-79.

Lim LY, Khor E, Ling CE. Effects of dry heat and saturated steam on the physical properties of chitosan. Journal of Biomedical Materials Research. 1999; 48:111-6. http:// dx.doi.org/10.1002/(SICI)1097-4636(1999)48:2<111::AI D-JBM3>3.0.CO;2-W

Maniglia-Ferreira C, Silva Junior J, Paula R, Feitosa J, Cortez D, Zaia A, Souza-Filho F. Brazilian gutta-percha points. Part I: chemical composition and x-ray diffraction analysis. Brazilian Oral Research. 2005; 19:193-7. PMid:16308607. http://dx.doi.org/10.1590/S1806-83242005000300007

Melker KB, Vertucci FJ, Rojas MF, Progulske-Fox A, Bélanger M. Antimicrobial efficacy of medicated root canal filling materials. Journal of Endodontics. 2006; 32:148-51. PMid:16427466. http://dx.doi.org/10.1016/j.joen.2005.10.032

Miao D, Blom D, Zhao H, Luan X, Chen T, Wu X, Song $\mathrm{N}$. The antibacterial effect of CMCTS-containing chewing gum. Journal of Nanjing Medical University. 2009; 23:69-72. http://dx.doi.org/10.1016/S1007-4376(09)60030-7

Minagawa T, Okamura Y, Shigemasa Y, Minami S, Okamoto Y. Effects of molecular weight and deacetylation degree of chitin/chitosan on wound healing. Carbohydrate Polymers, 2007; 67:640-4. http://dx.doi.org/10.1016/j. carbpol.2006.07.007

Mohire NC, Yadav AV. Chitosan-based polyherbal toothpaste: As novel oral hygiene product. Indian Journal of Dental Research. 2010; 21:380-4. PMid:20930349. http://dx.doi. org/10.4103/0970-9290.70808

Morrier JJ, Benay G, Hartmann C, Barsotti O. Antimicrobial activity of $\mathrm{Ca}(\mathrm{OH})_{2}$ dental cements: an in vitro study. Journal of Endodontics. 2003; 29:51-4. PMid:12540221. http:// dx.doi.org/10.1097/00004770-200301000-00014

No HK, Park NY, Lee SH, Meyers SP. Antibacterial activity of chitosans and chitosan oligomers with different molecular weights. Internationa Journal of Food Microbiology. 2002; 74:65-72. http://dx.doi.org/10.1016/ S0168-1605(01)00717-6

Opanasopit P, Aumklad P, Kowapradit J, Ngawhiranpat T, Apirakaramwong A, Rojanarata T, Puttipipatkhachorn S. Effect of salt forms and molecular weight of chitosans on in vitro permeability enhancement in intestinal epithelial cells (Caco-2). Pharmaceutical Development and Technology. 2007; 12: 447-55. PMid:17963144. http:// dx.doi.org/10.1080/10837450701555901

Petti S, Scully C. Polyphenols, oral health and disease: A review. Journal of Dentistry. 2009; 37: 413-23. PMid:19303186. http://dx.doi.org/10.1016/j. jdent.2009.02.003

Pignatello R, Basile L, Puglisi G. Chitosan glutamate hydrogels with local anesthetic activity for buccal application. Drug Delivery. 2009; 16:176-81. PMid:19514978. http:// dx.doi.org/10.1080/10717540902861267

Porporatto C, Bianco I, Riera C, Correa S. Chitosan induces different L-arginine metabolic pathways in resting and inflammatory macrophages. Biochemical and Biophysical Research Communications, 2003; 304:266-72. http://dx.doi. org/10.1016/S0006-291X(03)00579-5

Qin C, Gao J, Wang L, Zeng L, Liu, Y. Safety evaluation of short-term exposure to chitooligomers from enzymic preparation. Food and Chemical Toxicology. 2006; 44:855-61. PMid:16442198. http://dx.doi.org/10.1016/j.fct.2005.11.009

Raafat D, Von Bargen K, Haas A, Sahl HG. Insights in the mode of action of chitosan as an antibacterial compound. Applied and Environmental Microbiology. 2008; 74:3764-73. PMid:18456858 PMCid:2446574. http://dx.doi.org/10.1128/ AEM.00453-08

Rabea EI, Badawy ME, Stevens CV, Smagghe G, Steurbaut W. Chitosan as antimicrobial agent: applications and mode of action. Biomacromolecules. 2003; 4:1457-65. PMid:14606868. http://dx.doi.org/10.1021/bm034130m

Rao SB, Sharma CP. Use of chitosan as biomaterial: studies on its safety and hemostatic potential. Journal of Biomedical Materials Research. 1997; 34:21-28. http:// dx.doi.org/10.1002/(SICI)1097-4636(199701)34:1<21::AI D-JBM4>3.0.CO;2-P

Rhoades J, Roller S. Antimicrobial actions of degraded and native chitosan against spoilage organisms and laboratory media and foods. Applied and Environmental Microbiology. 2000; 66:80-6. http://dx.doi.org/10.1128/ AEM.66.1.80-86.2000 
Sano H, Shibasaki KI, Matsukubo T, Takaesu Y. Effect of chitosan rinsing on reduction of dental plaque formation. The Bulletin of Tokyo Dental College. 2003; 44:9-16. PMid:12772581. http://dx.doi.org/10.2209/tdcpublication.44.9

Sano H, Shibasaki KI, Matsukubo T, Takaesu Y. Effect of molecular mass and degree of deacetylation of chitosan on adsorption of Streptococcus sobrinus 6715 to saliva treated hydroxyapatite. The Bulletin of Tokyo Dental College. 2002; 43:75-82. PMid:12174667. http://dx.doi. org/10.2209/tdcpublication.43.75

Şenel S, Íkinci G, Kaș S, Yousefi-Rad A, Sargon MF, Hincal AA. Chitosan films and hydrogels of chlorhexidine gluconate for oral mucosal delivery. International Journal of Phamaceutics. 2000; 193:197-203. http://dx.doi.org/10.1016/ S0378-5173(99)00334-8

Silva H, Santos K, Ferreira E. Quitosana: derivados hidrossolúveis, aplicações farmacêuticas e avanços. Química Nova. 2006; 29:776-5. http://dx.doi.org/10.1590/ S0100-40422006000400026

Siqueira JF, Rôças I, Favieri A, Abad E, Castro AJR, Gahyva SM. Bacterial leakage in coronally unsealed root canals obturated with 3 different techniques. Ora Surgery Oral Medicine Oral Pathology Oral Radiology and Endodontology. 2000; 90:647-50. PMid:11077391. http:// dx.doi.org/10.1067/moe.2000.110412

Siqueira JF, Rôças I. Clinical implications and microbiology of bacterial persistence after treatment procedures. Journal of Endodontics. 2008; 34:1291-301. PMid:18928835. http:// dx.doi.org/10.1016/j.joen.2008.07.028

Spin-Neto R, Pavone C, Freitas RM, Marcantonio R, Marcantonio-Júnior E. Biomateriais à base de quitosana com aplicação médica e odontológica: revisão de literatura. Revista de Odontologia da UNESP. 2008; 37:155-61.

Sun T, Zhou D, Xie J, Mao F. Preparation of chitosan oligomers and their antioxidant activity. European Food Research and Technology. 2007; 225:451-6. http://dx.doi. org/10.1007/s00217-006-0439-1

Sun L, Du Y, Fan L, Chen X, Yang J. Preparation, characterization and antimicrobial activity of quaternized carboxymethyl chitosan and application as pulp-cap. Polymer. 2006; 47:1796-804. http://dx.doi.org/10.1016/j. polymer.2006.01.073

Sutherland IW. The biofilm matrix - an immobilized but dynamic microbial environment. Trends in Microbiology. 2001; 9: 222-7. http://dx.doi.org/10.1016/ S0966-842X(01)02012-1
Takahashi N. Microbial ecosystem in the oral cavity: metabolic diversity in an ecological niche and its relationship with oral diseases. International Congress Series. 2005; 1284:103-12. http://dx.doi.org/10.1016/j.ics.2005.06.071

Tarsi R, Muzzarelli R, Guzmàn C, Pruzzo C. Inhibition of Streptococcus mutans adsorption to hydroxyapatite by low-molecular-weigth chitosans. Journal of Dental Research. 1997; 76:665-72. PMid:9062560. http://dx.doi. org/10.1177/00220345970760020701

Tavaria F, Jorge M, Marchetti G, Souza V, Ruíz AL, Malcata X, Pintado M, Carvalho JE. Wound healing, anti-ulcerogenic, anti-inflammatory and anti-proliferative properties of chitosan. New Biotechnology. 2009; 25:S10. http://dx.doi. org/10.1016/j.nbt.2009.06.027

Ueno H, Nakamura F, Murakami M, Okumura M, Kadosawa T, Fijinaga T. Evaluation effects of chitosan for the extra-cellular matrix production by fibroblasts and the growth factors production by macrophages. Biomaterials, 2001a; 22:2125-30. http://dx.doi.org/10.1016/ S0142-9612(00)00401-4

Ueno H, Murakami M, Okumura M, Kadosawa T, Uede $\mathrm{T}$, Fijinaga T. Chitosan accelerates the production of osteopontin from polymorphonuclear leukocytes. Biomaterials, 2001b; 22:1667-73. http://dx.doi.org/10.1016/ S0142-9612(00)00328-8

Uysal T, Akkurt MD, Amasyali M, Ozcan S, Yagci A, Basak F, Sagdic D. Does a chitosan-containing dentifrice prevent demineralization around orthodontic brackets? Angle Orthodontist. 2011; 81:319-5. PMid:21208086. http://dx.doi.org/10.2319/062910-359.1

Verkaik MJ, Busscher HJ, Jager D, Slomp AM, Abbas F, Van der Mei HC. Efficacy of natural antimicrobials in toothpaste formulations against oral biofilms in vitro. Journal of Dentistry. 2011; 39:218-24. PMid:21195122. http://dx.doi.org/10.1016/j.jdent.2010.12.007

Wenjun L, Xuan J, Peihua X, Shiming C. Inhibitory effects of chitosan on superoxide anion radicals and lipid free radicals. Chinese Science Bulletin. 2002; 47:887-9. http:// dx.doi.org/10.1360/02tb9198

Xing R, Yu H, Liu S, Zhang W, Zhang Q, Li Z, Li P. Antioxidant activity of differently regioselective chitosan sulfates in vitro. Bioorganic and Medicinal Chemistry. 2005; 13:1573-7. PMid:15698774. http:// dx.doi.org/10.1016/j.bmc.2004.12.022

\footnotetext{
Autores

Freni Kekhasharú Tavaria*, Eduardo Manuel Costa, Maria Manuela Pintado

Centro de Biotecnologia e Química Fina, Universidade Católica Portuguesa, Rua Dr. António Bernardino de Almeida, 4200-072, Porto, Portugal.
} 